\title{
Null mutations of NEUROG3 are associated with delayed-onset diabetes mellitus
}

\author{
R. Sergio Solorzano-Vargas, ${ }^{1}$ Matthew Bjerknes, ${ }^{2}$ Jiafang Wang, ${ }^{1}$ S. Vincent $\mathbf{W u},{ }^{3,4}$ \\ Manuel G. Garcia-Careaga, ${ }^{5}$ Pisit Pitukcheewanont, ${ }^{6}$ Hazel Cheng, ${ }^{2}$ Michael S. German, ${ }^{7,8}$ \\ Senta Georgia, ${ }^{6}$ and Martín G. Martín ${ }^{1,9}$ \\ 'Division of Gastroenterology and Nutrition, Department of Pediatrics, Mattel Children's Hospital and David Geffen School \\ of Medicine at UCLA, Los Angeles, California, USA. '2Department of Medical Biophysics, Faculty of Medicine, University of \\ Toronto, Toronto, Ontario, Canada. ${ }^{3}$ Veterans Affairs Greater Los Angeles Healthcare System, Los Angeles, California, USA. \\ ${ }^{4}$ Department of Medicine, UCLA, Los Angeles, California, USA. ${ }^{5}$ Department of Pediatrics, Stanford Medicine, \\ Palo Alto, California, USA. 'Division of Endocrinology, Department of Pediatrics, Children's Hospital Los Angeles and \\ University of Southern California, Los Angeles, California, USA. ${ }^{7}$ Diabetes Center and ${ }^{8}$ Department of Medicine, UCSF, \\ San Francisco, California, USA. ${ }^{9}$ Eli and Edythe Broad Center of Regeneration Medicine and Stem Cell Research, Los \\ Angeles, California, USA
}

Biallelic mutations of the gene encoding the transcription factor NEUROG3 are associated with a rare disorder that presents in neonates as generalized malabsorption - due to a complete absence of enteroendocrine cells - followed, in early childhood or beyond, by insulin-dependent diabetes mellitus (IDDM). The commonly delayed onset of IDDM suggests a differential requirement for NEUROC3 in endocrine cell generation in the human pancreas versus the intestine. However, previously identified human mutations were hypomorphic and, hence, may have had residual function in pancreas. We report 2 patients with biallelic functionally null variants of the NEUROC3 gene who nonetheless did not present with IDDM during infancy but instead developed permanent IDDM during middle childhood ages. The variants showed no evidence of function in traditional promoter-based assays of NEUROC3 function and also failed to exhibit function in a variety of potentially novel in vitro and in vivo molecular assays designed to discern residual NEUROC3 function. These findings imply that, unlike in mice, pancreatic endocrine cell generation in humans is not entirely dependent on NEUROC3 expression and, hence, suggest the presence of unidentified redundant in vivo pathways in human pancreas capable of yielding $\beta$ cell mass sufficient to maintain euglycemia until early childhood.

Conflict of interest: The authors have declared that no conflict of interest exists.

Copyright: (ㄷ) 2020, American Society for Clinical Investigation.

Submitted: January 23, 2019 Accepted: November 21, 2019 Published: January 16, 2020.

Reference information: /CI Insight. 2020;5(1):e127657.

https://doi.org/10.1172/jci. insight.127657.

\section{Introduction}

NEUROG3 is a basic helix-loop-helix (bHLH) transcription factor that drives endocrine lineage generation in the gut, pancreas, and hypothalamus (1-3). Human neonates with biallelic dysfunctional NEUROG3 present clinically with enteric anendocrinosis (MIM:\#610370), characterized by generalized malabsorption and an absence of enteroendocrine cells (EECs) (4-6). As these children age, hypogonadotropic hypogonadism and short stature become evident (7), and at a variable age (from 20 days to more than 23 years of age), they develop insulin-dependent diabetes mellitus $(\operatorname{IDDM})(8,9)$.

An in vitro directed-differentiation protocol fails to generate any significant number of pancreatic endocrine cells from human pluripotent stem cells if NEUROG3 function is disabled by gene editing (10, 11). NEUROG3 deletion experiments in pigs $(3,12)$ and mice $(3)$ have similarly demonstrated failure of endocrine cell generation in the developing pancreas, resulting in a permanent neonatal diabetes mellitus (PNDM) phenotype. Such results have led to the conclusion that NEUROG3 is essential for human $\beta$ cell development. Hence, it has also been concluded that the NEUROG3 mutations affecting patients exhibiting delayed-onset IDDM (e.g., p.R107S) must be hypomorphic, displaying insufficient transactivating activity to enable generation of EECs in the gut, but nonetheless retain sufficient activity to initiate some minimal level of pancreatic endocrine differentiation during development $(8,11)$. 
Standard tests of the functional competence of human NEUROG3 variants have significant background activity, making it difficult to distinguish weak residual hypomorphic activity from effectively null activity (5). Thus far, tests have been limited to in vitro reporter and gel shift assays of mutant NEUROG3 interactions with a well-studied E-box (12) located in the immediate promoter region of neurogenic differentiation factor 1 (NEUROD1) and in vivo assessment of the level of NEUROD1 or glucagon expression driven by mutant NEUROG3 when expressed in Xenopus laevis or chicken embryos, respectively $(5,9)$.

NEUROG3's ability to repress the cell cycle offers an alternate assay of its functional competence (13). We recently found that expressing NEUROG3 in a human endocrine cell line induces cellular quiescence in a p21CIP1-dependent fashion, while prolonged expression induces cellular senescence in a p16INK4A-dependent manner (14). Furthermore, early NEUROG3-induced cellular quiescence is reversible by inhibition of PTEN, due to a reduction in steady-state NEUROG3 and p21CIP1 levels in BON4 cells and human intestinal enteroids.

Here, we describe and demonstrate the functional incompetence of 2 probands with homozygous severe nonsense mutations of NEUROG3. Importantly, in addition to enteric anendocrinosis, both had delayed-onset permanent IDDM, rather than the PNDM expected to arise if NEUROG3 is indeed essential for human pancreatic endocrine cell generation.

\section{Results}

Subjects' clinical characteristics. The 2 unrelated index cases are a male and female of Mexican-American descent, each the product of consanguineous union. They were both hospitalized within the first weeks after birth with severe diarrhea, weight loss, and dehydration. Both required a combination of parenteral and enteral nutrition for several years, with diarrheal symptoms that improved but persisted throughout their childhoods. Intestinal biopsies revealed that both children lacked EECs, while $\mathrm{Na}^{+}$glucose/galactose cotransporter (SLC5A1) expression was similar to that of controls (Figure 1E and our unpublished observations). IDDM began by 3 years of age in proband 1 and by 7 years of age in proband 2 . The probands are currently 18 and 14 years old, respectively. Detailed clinical information is provided in the Supplemental Clinical Information (supplemental material available online with this article; https://doi.org/10.1172/jci.insight.127657DS1).

Sequencing of the NEUROG3 gene. We sequenced the single coding exon of NEUROG3 from the 2 index cases and their biological parents.

Proband 1 has a homozygous deletion of cytosine at nucleotide 117 that results in a frame shift mutation beginning at amino acid 40 (Figure 1A). This variant, p.P39PfsX38, (hereafter referred to as $N E U$ $R O G 3^{N U L L}$ ) encodes a protein missing the nuclear localization, HLH, and activation (AD) domains, which suggested that it likely has no residual functional activity (Figure 1C).

Proband 2 has a homozygous insertion of cytosine at nucleotide 431 that results in a frame shift mutation beginning at amino acid 145 (Figure 1B). This variant, p.H144Pfs*94 (hereafter referred to as $N E U$ $R O G 3^{D N}$ ) retains the bHLH domain but lacks the protein's entire $\mathrm{AD}$, which suggested that it likely exhibits dominant-negative (DN) characteristics without residual functional activity (Figure 1C).

These variants were not previously observed in the Exome Aggregation Consortium (ExAC) database (http://exac.broadinstitute.org) of over 121,000 individuals and are therefore very rare (MAF $<0.0001 \%$ ) (data not shown).

Reduced pancreatic islets in a patient with NEUROG3 missense mutation. Our original report described a proband with a NEUROG3 ${ }^{\text {R107s }}$ missense mutation. This patient succumbed to central catheter sepsis prior to the onset of IDDM (5). Immunostaining of pancreas obtained at autopsy demonstrated both glucagon and insulin ${ }^{+}$cells, but the frequency of insulin ${ }^{+}$cells was nearly 5 -fold less than in age-matched controls (Figure 1D and data not shown).

Intracellular localization of FLAG-tagged recombinant NEUROG $3^{W T}, N E U R O G 3^{N U L L}$, and NEUROG $3^{D N}$ expressed in BON4 cells. To examine the functional consequences of these severe variants, we assessed their behavior in a homogenous isolate of the endocrine-derived human BON cell line, herein referred to as BON4 cells (14). BON4 cells do not express endogenous NEUROG3 and are therefore entirely responsive to heterologous expression. BON4 cells transduced with lentiviruses that constitutively express a C-terminus FLAG-tagged sequence encoding WT (NEUROG $3^{\mathrm{WT}}$ ), the 2 previously described missense variants (NEUROG3 ${ }^{\mathrm{R} 93 \mathrm{~L}}$ and NEUROG $\left.3^{\text {R107S }}\right)(5,15)$, or the 2 potentially novel variants (NEUROG3 ${ }^{\text {NULL }}$ and NEUROG3 ${ }^{\mathrm{DN}}$ ) were used to assess the intracellular localization of each variant (Figure 1C). Cells transduced with NEUROG3WT, NEUROG $3^{\mathrm{R} 93 \mathrm{~L}}$, or NEUROG $3^{\mathrm{R} 107 \mathrm{~S}}$ appropriately localized exclusively in the nucleus, while the NEUROG3 $3^{\mathrm{NULL}}$ 
A

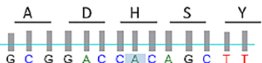

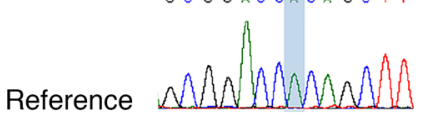
$\stackrel{A}{D} \stackrel{P}{\mathrm{Q}} \stackrel{\mathrm{L}}{\mathrm{H}}$
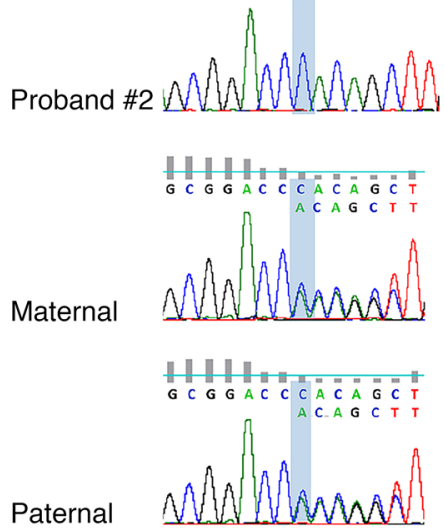

c.431insC; p.H144PfsX94
B

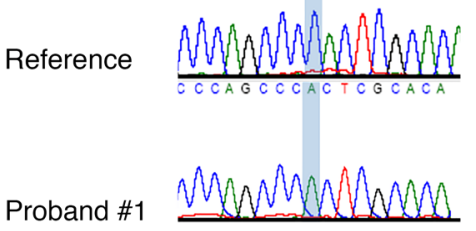

c.117delC;P39PfsX38

\section{C}

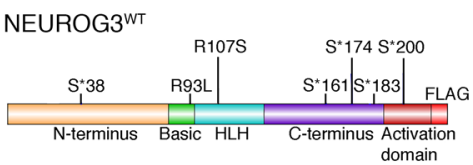

NEUROG3 ${ }^{\text {DN }}$

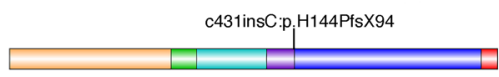

NEUROG3 ${ }^{\text {NULL }}$

c177delC.p.P39PfsX38
D

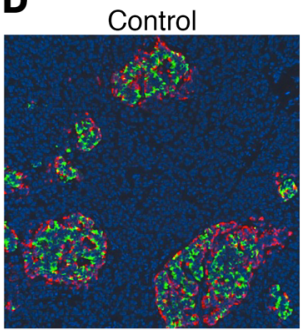

E

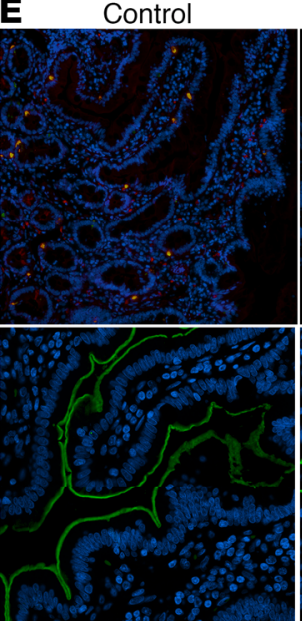

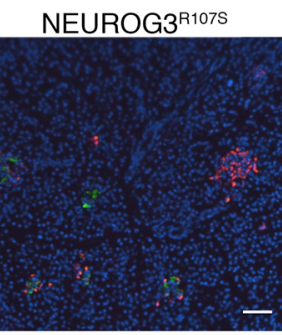

NEUROG3 ${ }^{\text {DN }}$

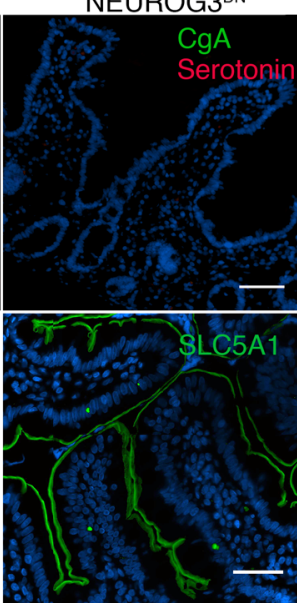

Figure 1. Proband NEUROG3 variants and histology. (A) Diagram comparing the NEUROG3 Sanger sequence of reference and proband 1, demonstrating a biallelic deletion of a cytosine at position c.117, resulting in the c.117delC or p.P39PfsX38 variant. (B) Sanger sequencing results for proband 2 and her 2 parents, demonstrating a homozygous insertion of a cytosine at position 431, resulting in a frame shift mutation, resulting in the c.431insC or p.H144PfsX94 variant. (C) Schematic diagram of NEUROG ${ }^{W T}$ showing the location of its basic (green), HLH (aqua blue), and AD domains (deep red). The C-terminal FLAC domain (red) serves as a NEUROC3 marker in our experiments. The structure of the NEUROC $3^{\text {DN }}$ variant shows the frame shift induced deletion of the $A D$ domain and its replacement with aberrant segment (blue). Diagram of NEUROG $3^{\text {NULL }}$ showing location of the variant and an aberrant segment (gray). (D) Pancreatic autopsy sample from the age-matched control and the original proband (p.R107S) stained with anti-glucagon (red) and anti-insulin (green) antibodies. Scale bar: $100 \mu \mathrm{m}$. (E) Intestinal biopsy from control and NEUROC3 ${ }^{\mathrm{DN}}$ samples stained with anti-Chga (green), serotonin (red), and the $\mathrm{Na}^{+}$ glucose/galactose cotransporter (SLC5A1). Scale bar: $100 \mu \mathrm{m}$.

variant was located diffusely across the cytoplasm and nucleus, and the NEUROG $3^{\mathrm{DN}}$ mutant appears especially concentrated in some subnuclear structures (Supplemental Figure 1A).

NEUROG $3^{\text {NULL }}$ and NEUROG $3^{D N}$ fail to activate the promoter of the NEUROG3 target gene NEUROD1. NEUROG3 activates expression of its downstream target NEUROD1 via interaction with an E-box in the NEUROD1 promoter $(5,16)$. We assessed the ability of each NEUROG3 variant to activate this promoter. We constructed a separate mammalian expression plasmid for each of NEUROG3 ${ }^{\mathrm{WT}}$, NEUROG3 ${ }^{\text {R93L }}$, NEUROG3 ${ }^{\text {R107s }}$, NEUROG3 ${ }^{\text {NULL }}$, and NEUROG3 ${ }^{\text {DN }}$. Each construct was cotransfected into BON4 cells with a plasmid containing the immediate promoter region of NEUROD1 driving a luciferase reporter (5). The NEUROG $3^{\mathrm{WT}}$ construct induced a pronounced activation of the reporter, the NEURO$\mathrm{G}^{\mathrm{R} 93 \mathrm{~L}}$ and NEUROG3 ${ }^{\mathrm{R} 107 \mathrm{~s}}$ variants induced only a mild response, and the responses to NEUROG3 ${ }^{\text {NULL }}$ and NEUROG $3^{\mathrm{DN}}$ were not significantly different from those induced by an empty expression vector control (Figure 2A). The fidelity of NEUROG3 ${ }^{\mathrm{WT}}$ in BON4 cells was confirmed by its failure to upregulate the non-NEUROG3 target - SLC5A1 - via the E-box element in its proximal promoter (ref. 17 and Supplemental Figure 1B). Western blotting confirmed that similar levels of transfected proteins were expressed (Supplemental Figure 1D).

We also tested each NEUROG3 variant's interaction with the NEUROD1 promoter E-box by performing electrophoretic mobility shift analysis after incubation of the promoter sequence with nuclear extracts isolated from BON4 cells previously transfected with the plasmids containing the various NEUROG3 variants. A prominent gel shift occurred only with extracts isolated from cells transfected with NEUROG3 ${ }^{\text {WT }}$ (Supplemental Figure 1E).

In summary, the NEUROG $3^{\mathrm{NULL}}$ variant induced only background levels of activation of the $N E U$ $R O D 1$ promoter and showed no evidence of direct interaction with its E-box. 
A

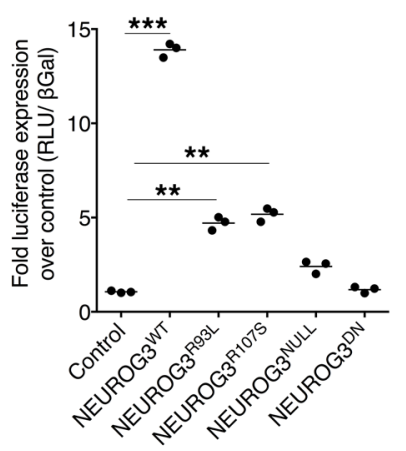

B

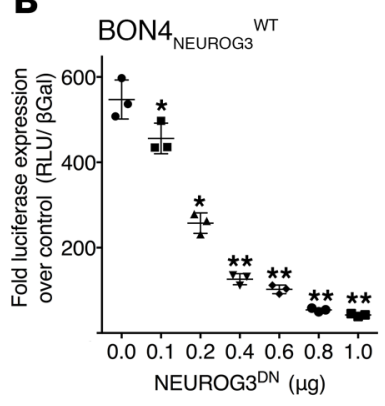

C

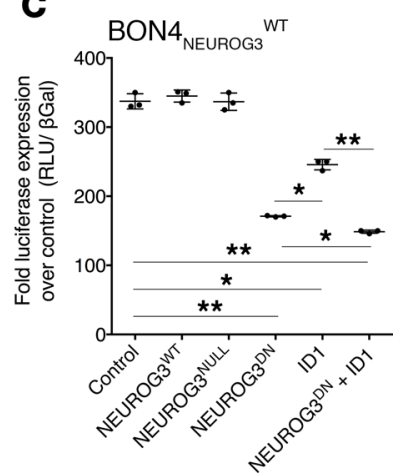

Figure 2. Human NEUROD1 promoter activity altered by NEUROG3 variants in BON4 cells. (A) Luciferase activity from cells cotransfected with reporter construct and either control or various NEUROG3 variants in a mammalian expression vector and luciferase normalized with $\beta$-Gal. (B) Promoter activity of cells transduced with constitutively active NEUROC ${ }^{\text {WT }}$ lentivirus and cotransfected with luciferase construct and increasing amounts of the NEUROC $3^{\mathrm{DN}}$ variant. (C) Promoter activity of cells transduced with NEUROC $3^{\text {WT }}$ and cotransfected with increasing amounts of specified expression vectors. ${ }^{*} P<0.05$, ${ }^{* *} P<0.01$, ${ }^{* *} P<0.005$, 1-way ANOVA with Dunnett's multiple comparisons test.

NEUROG $3^{D N}$ can function as a DN. NEUROG $3^{\text {DN }}$ has intact DNA-recognition and -binding domains but lacks the $\mathrm{AD}$ domain. Thus, its failure to activate the NEUROD1 promoter is consistent with the idea that it exhibits DN potential. To assess this possibility, BON4 cells were transduced with a NEUROG3 ${ }^{\mathrm{WT}}$ lentivirus, generating $B O N 4_{\text {NEUROG } 3} W T$ cells, which were then cotransfected with a NEUROD1 promoter luciferase reporter construct and increasing amounts of a vector expressing NEUROG $3^{\mathrm{DN}}$. The NEUROG$3^{\mathrm{WT}}$-driven reporter expression decreased in proportion to the amount of transfected NEUROG ${ }^{\mathrm{DN}}$ variant (Figure 2B). Similarly, BON4 cells were transduced with a NEUROG $3^{\mathrm{DN}}$-expressing lentivirus, generating $B O N 4_{\text {NEUROG } 3} D N$ cells, which were then cotransfected with increasing concentrations of NEUROG3 ${ }^{\text {WT }}$ plasmid and the $N E U R O D 1$ reporter construct. In this case reporter activity remained low and was only mildly enhanced in cells transfected with the largest amount of the NEUROG3 ${ }^{\mathrm{WT}}$ vector (Supplemental Figure 1C; compare with the $\sim 10$-fold increase in Figure $2 \mathrm{~A}$ ).

Next, we compared the DN inhibitory activity of the NEUROG $3^{\mathrm{DN}}$ variant with ID1, a well-characterized DN inhibitor of bHLH transcriptional activation (18). BON4 NEUROG 3 WT cells were cotransfected with the same amount of the NEUROD1 luciferase reporter and increasing amounts of ID1, NEUROG3 ${ }^{\mathrm{DN}}$, or both. The data suggest that the NEUROG $3^{\mathrm{DN}}$ variant is more potent than ID1, but the 2 in combination synergistically attenuated NEUROG $3^{\mathrm{WT}}$-induced expression of NEUROD1 promoter activity (Figure 2C). Overall, these data support the notion that the NEUROG $3^{\mathrm{DN}}$ protein can act in a DN fashion to inhibit NEUROG3-induced activity on the NEUROD1 promoter.

The NEUROG GNULL and NEUROG $3^{D N}$ variants fail to repress cellular proliferation. NEUROG3 is known to repress proliferation in BON4 cells (14), offering an independent assay of the functional competence of the NEUROG3 variants. To test for repression, we transduced BON4 cells with lentiviruses encoding each $N E U$ $R O G 3$ variant. The corresponding transduced cells were denoted as follows: $B O N 4_{\mathrm{NEUROG}_{3}}{ }^{W T}, B O N 4_{\mathrm{NEUROG}} D N$, $B O N 4_{\mathrm{NEUROG} 3}{ }^{N U L L}, B O N 4_{\mathrm{NEUROG} 3} R 93 L$, and $B O N 4_{\mathrm{NEUROG} 3} R 107 S$. We assessed proliferation in the transduced cells by Ki67 staining as well as the MTT and FACS assays. As expected from previous observations (14), proliferation of $B O N 4_{\mathrm{NEUROG}^{3}} W T$ cells was repressed. Thus, $\mathrm{Ki} 67^{+}$cells were rarely seen among transduced $\left(\mathrm{GFP}^{+}\right)$ cells, and there was reduced MTT activity and an increase of cells in the $\mathrm{G}_{0} / \mathrm{G}_{1}$ phase of the cell cycle (Figure 3). In contrast, the measures of proliferation in the $B O N 4_{\mathrm{NEUROG}_{3}}{ }^{N U L L}$ and $B O N 4_{\mathrm{NEUROG}^{3}} D N$ cells were indistinguishable from those in GFP controls (Figure 3 and Table 1). Finally, we examined whether NEUROG3 ${ }^{\text {DN }}$ attenuates the ability of $B O N 4_{\text {NEUROG } 3} W T$ to induce cell cycle arrest. Indeed, transduction of NEUROG3 ${ }^{\mathrm{DN}}$ was able to entirely inhibit the NEUROG $3^{\mathrm{WT}}$-induced cell cycle arrest (Figure 4A).

The NEUROG3-induced changes in the cell cycle are mediated in part by binding an E-box element in the immediate promoter region of the p21CIP1 gene (19). Therefore, it was important to assess whether NEUROG $3^{\mathrm{DN}}$ attenuated NEUROG $3^{\mathrm{WT}}$-mediated induction of the $\mathrm{p} 21 \mathrm{CIP1}$ promoter. We transiently cotransfected BON4 cells with a human $\mathrm{p} 21$ CIP1 promoter luciferase reporter vector and a mammalian expression vector containing either a NEUROG $3^{\mathrm{WT}}$ or NEUROG $3^{\mathrm{DN}}$ insert. Increasing amounts of NEUROG3 ${ }^{\mathrm{WT}}$ augmented 
promoter activity, whereas the NEUROG $3^{\mathrm{DN}}$ variant failed to enhance activity (Figure 4B). To assess whether the NEUROG $3^{\mathrm{DN}}$ variant could completely attenuate NEUROG $3^{\mathrm{WT}}$-induced expression of the $\mathrm{p} 21$ ICIP1 promoter, we transfected $B O N 4_{\mathrm{NEUROG} 3} W T$ cells with the $\mathrm{p} 21 C I P 1$ promoter luciferase reporter vector and increasing amounts of the NEUROG $3^{D N}$ expression vector. We observed that NEUROG $3^{\mathrm{WT}}$-driven $\mathrm{p} 21 C I P 1$ promoter activity decreased in proportion to the quantity of transfected NEUROG3 $3^{\mathrm{DN}}$ vector (Figure $4 \mathrm{C}$ ).

Overexpression of NEUROG $3^{D N}$ attenuates the ability of NEUROG3WT to induce cellular senescence in BON4 cells. Another test of the functional competence NEUROG3 variants arises from the observation that overexpression of NEUROG3 in BON4 cells results in a time-dependent increase in p16INK4A expression, with the consequent induction of cellular senescence (14). The NEUROG3 variant transduced BON4 cell lines were examined for evidence of cellular senescence at 5 and 10 days of culture by staining for senescence-associated $\beta$-galactosidase (SA $\beta$-Gal), a marker of cellular senescence (20). BON4 $4_{\text {NEUROG } 3}{ }^{W T}$ cells exhibited robust SA $\beta$-Gal staining by 10 days, while $B O N 4_{\mathrm{NEUROG} 3} D N, B O N 4_{\mathrm{NEUROG} 3}{ }^{N U L L}, B O N 4_{\mathrm{NEURO}}$ ${ }_{\mathrm{G} 3} R 93 \mathrm{~L}$, and $B O N 4_{\mathrm{NEUROG} 3} R 107 \mathrm{~S}$ cell were indistinguishable from control cells (Figure $5 \mathrm{~A}$ ). We quantified SA $\beta$-Gal activity by lysing the cells and measuring the lysate's absorbance at $405 \mathrm{~nm}$. While $B O N 4_{\mathrm{NEU}}$ ${ }_{\text {ROG }}{ }^{W T}$ cells exhibited a 12 -fold increase in SA $\beta$-Gal levels by 15 days, the levels in BON4 ${ }_{\text {NEUROG } 3}{ }^{N U L L}$ and $B O N 4_{\text {NEUROG } 3} D N$ cells were indistinguishable from those in control cells (Figure 5B). When $B O N 4_{\mathrm{NEUROG}_{3}}{ }{ }$ cells were cotransduced with NEUROG $3^{\mathrm{DN}}$, SA $\beta$-Gal activity was strongly repressed, indicating that the cellular senescence induced by NEUROG $3^{\text {WT }}$ had been inhibited (Figure 5B). These findings demonstrate that NEUROG $3^{\mathrm{NULL}}$ and NEUROG $3^{\mathrm{DN}}$ lack the cellular senescence-inducing activity exhibited by NEUROG $3^{\text {WT }}$. Furthermore, NEUROG $3^{\text {DN }}$ attenuates the cellular senescence induced by NEUROG $3^{\mathrm{WT}}$, again indicating that NEUROG3 ${ }^{\mathrm{DN}}$ acts as a DN.

NEUROG $3^{N U L L}$ and NEUROG $3^{D N}$ variants fail to induce the endocrine phenotype exhibited by BON4 cells expressing NEUROG $3^{W T}$. The NEUROG3 ${ }^{\mathrm{WT}}$-induced cell cycle arrest in BON4 cells is known to be accompanied by increased expression of various EEC markers, including CHGA and PCSK1 (14). Therefore, we assayed the BON4 cells transduced with NEUROG3 ${ }^{\mathrm{WT}}$, NEUROG3 ${ }^{\mathrm{NULL}}$, NEUROG3 ${ }^{\mathrm{DN}}$, and NEUROG3 ${ }^{\mathrm{WT}}+$ NEUROG $3^{\text {DN }}$ for expression of these EEC markers. CHGA and PCSK1 were detectable by Western blot in NEUROG $3^{\text {WT }}$-transduced cells by 5 days, whereas they remained undetectable in NEUROG3 ${ }^{\text {NULL }}$-transduced cells (Figure 6A). The rapid and sustained expression of endocrine markers exhibited by BON4 ${ }_{\mathrm{NEU}}$ ${ }_{\text {ROG3 }} W T$ cells was strongly attenuated by cotransducing with NEUROG3 ${ }^{\text {DN }}$ (Figure 6). Interestingly, faint expression of $C H G A$ and PCSK1 was detectable in NEUROG3 ${ }^{\text {DN }}$ cells. We confirmed these findings using RT-qPCR for NEUROG3, CHGA, and PCSK1. Expression levels in BON4 ${ }_{\mathrm{NEUROG} 3}{ }^{N U L L}$ cells were always significantly less than those in $B O N 4_{\mathrm{NEUROG} 3}{ }^{D N}$ cells. (Figure 6, B-D).

NEUROG $3^{\text {NULL }}$ fails to induce the endocrine phenotype in Neurog $3^{\text {null }}$ murine enteroids. Intestinal enteroids grown from 0-day-old WT (Neurog $3^{w t}$ ) mouse pups exhibited abundant expression of the EEC marker Chga, while similar enteroids grown from Neurog3-null (Neurog3 ${ }^{\text {null }}$ ) pups did not (Figure 7, A-C, G, and H). We next tested whether transducing murine enteroids with lentiviruses encoding human NEUROG $3^{W T}$ enforces an EEC program. Neurog $3^{\text {wt }}$ and Neurog $3^{\text {null }}$ enteroids displayed greatly increased Chga expression 7 days after transduction (Figure 7, B, C, G, and H). Thus, the induction of Chga expression in Neurog $3^{\text {null }}$ enteroids provides a sensitive assay for NEUROG3 functional competence. We next applied this assay to assess the activity of the NEUROG3 ${ }^{\mathrm{NULL}}$ variant. Transducing Neurog $3^{\text {null }}$ enteroids with a lentivirus encoding NEUROG3 ${ }^{\text {NULL }}$ induced no change in Chga expression (Figure 7, C and G), confirming in vivo that the NEUROG3 ${ }^{\text {NULL }}$ variant identified in proband 1 exhibits no NEUROG3 activity.

NEUROG $3^{\text {DN }}$ represses endogenous NEUROG3 activity in human enteroids. We have consistently found that NEUROG $3^{\mathrm{DN}}$ represses NEUROG3 activity. We now ask whether it will do so in the context of human intestinal epithelium. Our group and others have described methods to grow human intestinal epithelium that continuously generates each of the differentiated epithelial cell lineages, including the NEUROG3-dependent EEC lineage $(21,22)$.

Next, WT human intestinal enteroids were transduced with a lentivirus expressing NEUROG $3^{D N}$. NEU $R O G 3^{D N}$ expression significantly decreased the expression of the NEUROG3-dependent endocrine markers (CHGA), compared with the expression observed in untransduced enteroids (Figure 7, D, I, and J), confirming that NEUROG3 ${ }^{\mathrm{DN}}$ acts a DN factor that impairs NEUROG3-dependent differentiation of EECs.

Native enteroids from a DN subject produce NEUROG3 as well as reduced EEC markers. To assess native characteristics in nontransduced enteroids, we measured the expression of EEC markers in small bowel enteroids grown from proband 2 carrying the NEUROG $3^{\text {DN }}$ variant compared with WT enteroids. Control enteroids 
A Contro
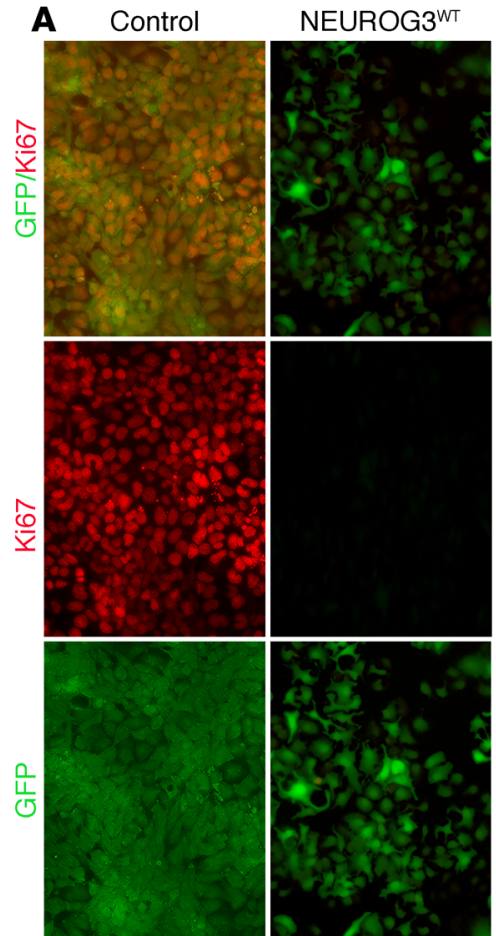

C

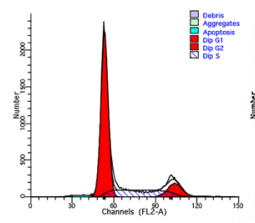

Control

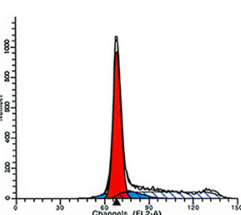

NEUROG3 ${ }^{W T}$
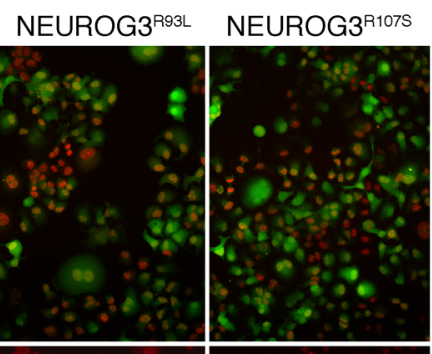

NEUROG3 ${ }^{\text {NULL }}$

NEUROG3 ${ }^{\mathrm{DN}}$
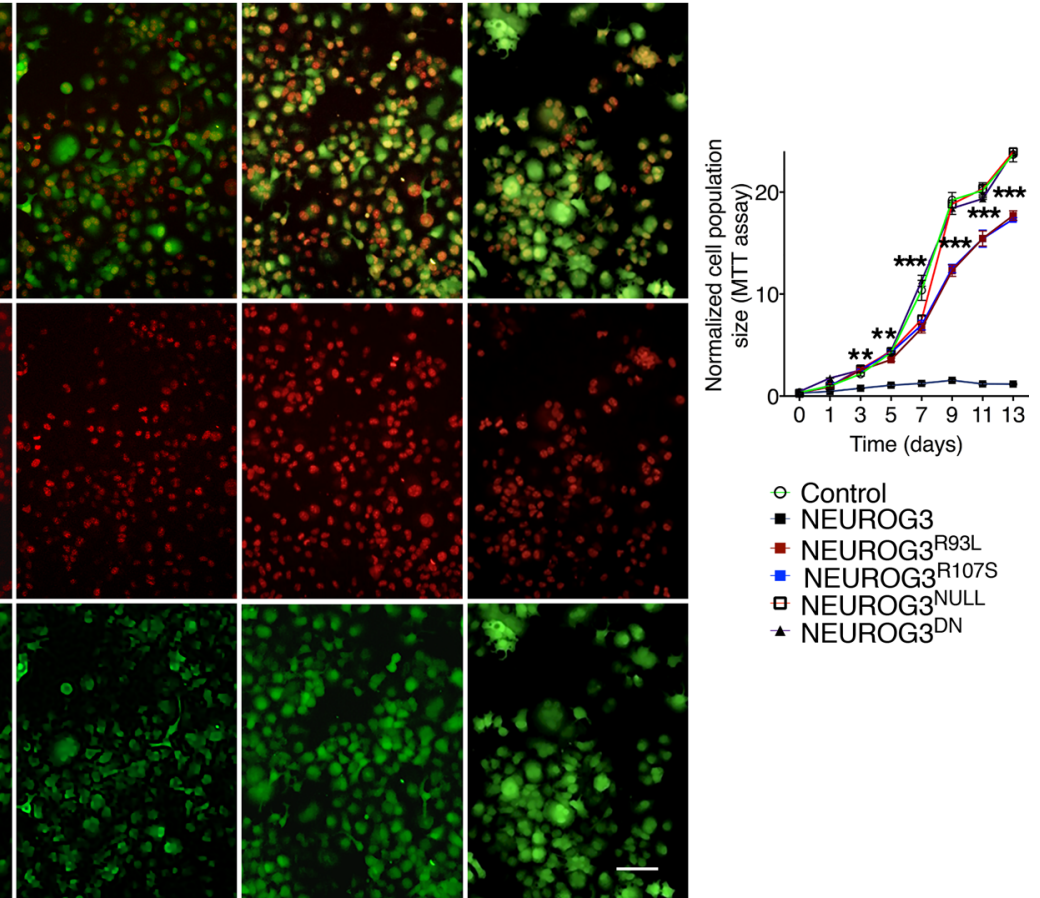

- Control

- NEUROG3

- NEUROG3 ${ }^{\mathrm{R} 93 \mathrm{~L}}$

- NEUROG3R107S

- NEUROG3 ${ }^{\text {NULL }}$

- NEUROG3 ${ }^{\mathrm{DN}}$

Figure 3. Proliferation of cells transduced with control or several NEUROG3 variants in BON4 cells. (A) Representative images of GFP and Ki67 staining. Scale bar: $100 \mu \mathrm{m}$. (B) MTT analysis of cells transduced 5 days earlier with constitutively active lentiviruses expressing NEUROG3 variants. (C) Representative FACS analysis of cells transduced with the various lentiviruses performed on day 5 . Shown are a representative histogram and the result of 3 separate experiments performed in triplicate. ${ }^{* *} P<0.01$, ${ }^{* *} P<0.005,2$-way ANOVA and 2-way Tukey's multiple comparisons.

expressed abundant CHGA and PCSK1; however, CHGA and PCSK1 were significantly reduced in the NEUROG3 $3^{\text {DN }}$ enteroid sample (Figure 7E). It should be noted that protein levels of the NEUROG3 ${ }^{\text {DN }}$ variant were comparable to NEUROG3 isolated from control sample. Finally, we assessed relevant transcript levels in control and NEUROG3 ${ }^{\text {DN }}$ enteroids, and as expected, CHGA and NEUROD1 mRNA levels were reduced in the enteroids from proband 2 when compared with control (Figure 7F). We also found that expression levels of related bHLH transcription factors (i.e., NEUROG1, NEUROG2, and NEUROD2) were not significantly elevated and therefore were not compensating for the loss of NEUROG3 function.

\section{Discussion}

Current understanding of the role of NEUROG3 in generating endocrine cells in humans is based on the clinical, pathologic, and metabolic abnormalities observed in children with biallelic mutations impairing NEUROG3 function.

The timing of the onset of diarrheal symptoms appears to be highly similar in children with various forms of enteric endocrinopathy or other enterocyte enteropathies $(5,6)$. In sharp contrast, the age of onset of IDDM varies widely in children with biallelic NEUROG3 variants (refs. 5, 23 and Supplemental Table 1). When we first described 3 children with missense mutations of NEUROG3 we postulated that unlike in mice, pancreatic endocrine cell fate in humans might be redundant with other transcription factor(s) since the surviving children were older than 8 years of age when they were first diagnosed with persistent IDDM (5). Others subsequently postulated that the failure of these subjects to develop persistent glucose intolerance was secondary to the hypomorphic nature of the variants that were described (24). Since then, 
Table 1. The effect of various NEUROG3 variants on cell cycle, apoptosis, and Ki67 staining in BON4 cells

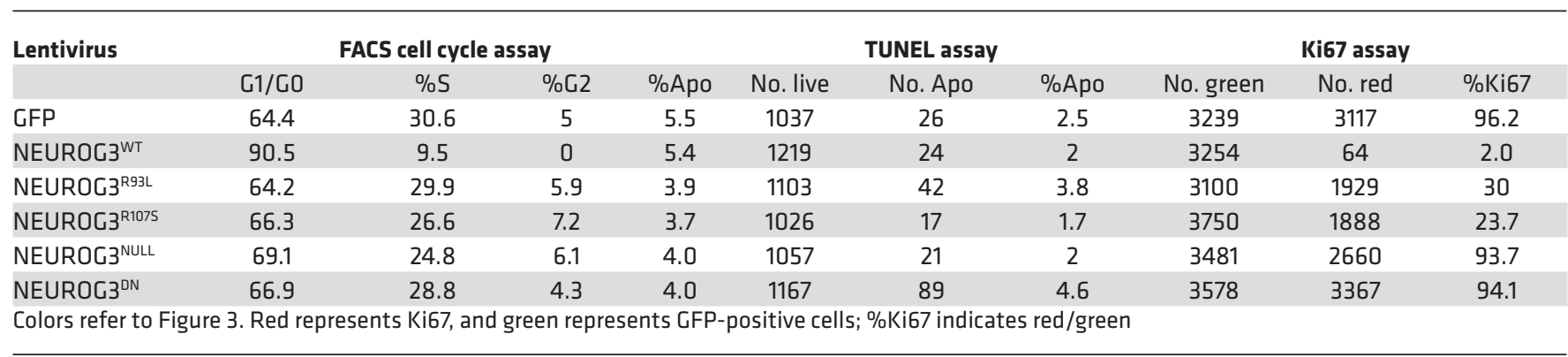

several new cases have been described where the age of onset varies from 20 days (9) to more than 23 years of age $(8,9,25)$; some of the younger patients may have developed hyperglycemia that was in part secondary to the use of glucocorticoids and macrolide calcineurin inhibitor (tacrolimus), administered to treat an incorrectly diagnosed autoimmune enteropathy (25). Interestingly, even siblings with identical homozygous NEUROG3 mutations (R107S) had dramatically disparate age of onset of persistent diabetic symptoms, suggesting that environmental or epigenetic factors may influence the age of onset of IDDM (ref. 8 and Supplemental Table 1). But this could all be attributed to some poorly understood residual NEUROG3 function present in these potentially hypomorphic variants.

Therefore, the fact that NEUROG $3^{N U L L}$ - the entirely nonfunctional nonsense variant of the NEUROG3 gene described here - results in delayed-onset IDDM, characteristic of the previously described hypomorphic variants, implies that some as-yet undescribed mechanism(s) exists for the generation of insulin-producing cells in the absence of NEUROG3 function. At least 3 possibly interacting classes of such potential mechanisms seem plausible and should form the basis of future studies:

NEUROG3-independent pathways capable of generating some pancreatic endocrine cells. Among the transcription factors known to be important for islet cell development, only RFX6 is consistently associated with both PNDM and malabsorptive diarrhea in a disorder named Mitchell-Riley syndrome (26). In this disorder, hyperglycemia begins immediately after birth and requires continuous insulin infusion - a phenotype distinct from that seen in most children with NEUROG3 mutations. Thus, RFX6 appears to be nonredundant with other factor(s) required for islet cell formation in humans and mice $(26,27)$. Indeed, genetic complementation studies in a novel murine Neurog $3^{\text {null }}$ pancreatic organ culture model suggest that Rfx6 and NeuroD1 stimulate endocrine cell fate in the absence of Neurog3 (28). Furthermore, assessment of the NEUROG3 ${ }^{\text {NULL }}$ variant failed to induce islet cell formation in a murine model system (28). But the role of NEUROG3 in human islet development remains uncertain. Notably, a human embryonic stem cell line, in which both NEUROG3 alleles had been disrupted by CRISPR/Cas9 targeting, entirely failed to generate pancreatic endocrine cells (11), seemingly demonstrating that NEUROG3 is essential for islet development, with the important proviso that the experiment was reliant on induction of differentiation by a standard in vitro differentiation induction protocol that may not enable alternate pathways available in the context of an intact embryo. However, others have been successful in generating a limited number of pancreatic endocrine cells in targeted NEUROG3 ablation studies that used different differentiation protocol (10). Production of pluripotent stem cell lines from patients with NEUROG3-dependent enteric anendocrinosis could help to resolve this. If such NEUROG3 variant stem cells generated $\beta$ cells, that would be convincing evidence of hypomorphic NEUROG3 function. Conversely, if $\beta$ cells are not generated from stem cells produced from patients displaying clear evidence of $\beta$ cell function, then the in vitro differentiation protocol would need to be reassessed.

Ectopic NEUROG3-independent (i.e., extraendocrine pancreas) sources of sufficient insulin to sustain the child. An example of such a source is the thymus. The neonatal thymus makes proinsulin as part of self-tolerance mechanisms, and this subsides with thymic involution in early childhood (29). However, it seems unlikely that this is the source of insulin that serves to modulate glucose levels in prediabetic NEUROG3 probands. "In the murine thymus, all pancreatic hormones (i.e., insulin, glucagon, and somatostatin) are expressed (30) at levels that are approximately 10,000-fold lower than in the pancreas (31) and are not modulated by metabolic modifications (32)." Nor is there any evidence that the thymus elaborates the complex cellular machinery necessary to process and secrete functional mature insulin in a glucose-responsive manner. Fur- 
A

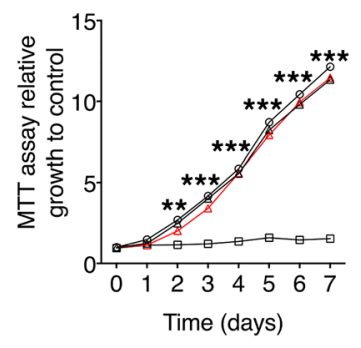

B

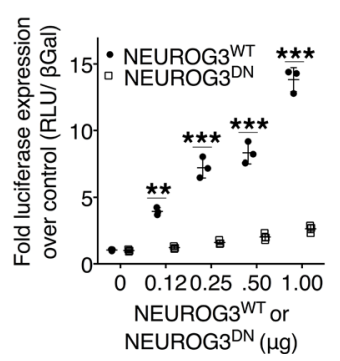

C

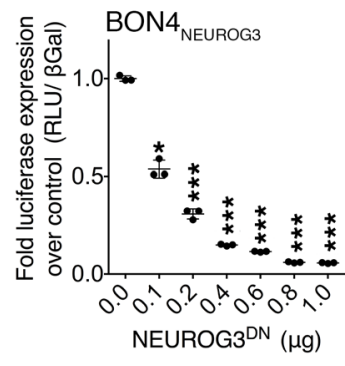

Figure 4. NEUROG ${ }^{\text {DN }}$ attenuates NEUROG $3^{\text {WT }}$ action in BON4 cells. (A) MTT analysis of cells transduced with constitutively active lentiviruses expressing control-IRES, NEUROG $3^{W T}$, and/or NEUROG ${ }^{\text {DN }}$ assessed daily for a week. (B) Cells cotransfected with the human $p 21$ promoter $(-592 /+41)$ construct and various amounts of either NEUROC $3^{\text {WT }}$ or NEUROC $3^{\text {DN }}$; luciferase normalized with $\beta$-Gal was measured 3 days later. (C) Cells transfected with $p 21$ promoter and transduced with NEUROC $3^{\mathrm{WT}}$ and cotransfected with increasing concentrations of NEUROG $3^{\mathrm{DN}}$ and luciferase as in B. (A and $\mathbf{B}){ }^{* *} P<0.01,{ }^{* *} P<0.005$, 2-way ANOVA and 2-way Tukey's multiple comparisons. (C) ${ }^{*} P<0.05,{ }^{* *} P<0.01,{ }^{* *} P$ $<0.005,1$-way ANOVA with Dunnett's multiple comparison.

thermore, the RFX6-null patients discussed above had diabetes mellitus at birth, arguing against the existence of an independent ectopic source of insulin in the neonate (unless RFX6 has a role in determining ectopic insulin transcription/processing, whereas the lineage factor NEUROG3 does not).

Spontaneous reverting mutations in NEUROG3 that reestablish some degree of NEUROG3 function in a pancreatic progenitor, enabling its resulting $N E U R O G 3^{+}$subclone to generate a limited population of endocrine cells sufficient to sustain the child. This seems improbable at first glance, but perhaps reversion-free NEUROG3 nulls succumb as neonates and hence go undiagnosed. The intestinal epithelium offers a simple test of the idea. If reverting mutations of the NEUROG3 locus occur with sufficient frequency to be a significant factor in pancreatic endocrine development, then this should manifest in the intestine as scattered crypts containing EECs (due to intestinal stem cells incurring reverting mutations, leading to a clone capable of generating EECs). Such EEC-containing crypts have not been seen, suggesting that the rate of reverting mutations is very low.

It is noteworthy that neither diabetes mellitus nor malabsorption have been reported in NEUROG3 heterozygous parents or siblings, including the parents of the index cases reported here. This is an especially interesting aspect of the NEUROG $3^{D N}$ family, because expression of the mother's DN variant allele would be expected to impair the function of her only normal NEUROG3 allele, implying that her progenitors experienced unusually low levels of NEUROG3 functional activity. This observation supports current understanding that low levels of NEUROG3 expression result in generation of sufficient pancreatic $\beta$ cells and EECs to avoid disease.

Frameshift mutations can induce nonsense-mediated mRNA decay (NMD) mechanisms, which could reduce the quantity of transcript and protein from the variant allele and, thereby, silence the DN effect in a heterozygote state (33). However, in the case of the NEUROG $3^{D N}$ variant, we show that the native protein in the patient's own enteroids sample is comparable in quantity in enteroids isolated from a control sample (Figure 7E). These findings and the absence of a concomitant increase in related bHLH transcription factors (Figure 7F) argue against the presence of NMD in our frameshift mutations. Furthermore, since $N E U$ ROG3 is encoded by a single exon, our results are consistent with other studies that have shown that NMD requires at least 1 intron with both donor and acceptor splice sites downstream of the nonsense codon (34).

It should also be pointed out that NEUROG $3^{\mathrm{DN}}$ induced marginal PCSK1 expression in BON4 cells and in the patient's native enteroids (despite an absence of frank EECs), the implications of which are unclear.

In mice, Neurog3 is expressed in endocrine pancreas development (30) during 2 fetal transitions that are associated with specific short bursts of Neurog3 expression (35). During the early phase (E8.5-E10.5) Neurog 3 induces a cluster of $\alpha$ cells, while the late phase (E12.5-E15) leads to the generation of primarily $\beta$ and $\delta$ cells $(35,36)$. Postnatally, the endocrine cell mass undergoes a proliferative phase that is independent of Neurog3 expression (37). In the intestine, Neurog 3 expression occurs continuously throughout late fetal and postnatal life and is limited to endocrine precursor cells derived from crypt base columnar cells, the intestinal epithelial stem cells (38). Moreover, Neurog $3^{+}$progenitor cells are either slowly cycling or postmitotic and express endocrine markers (2). Neurog $3^{\text {zull }}$ pups expire during within the first day of life and are 


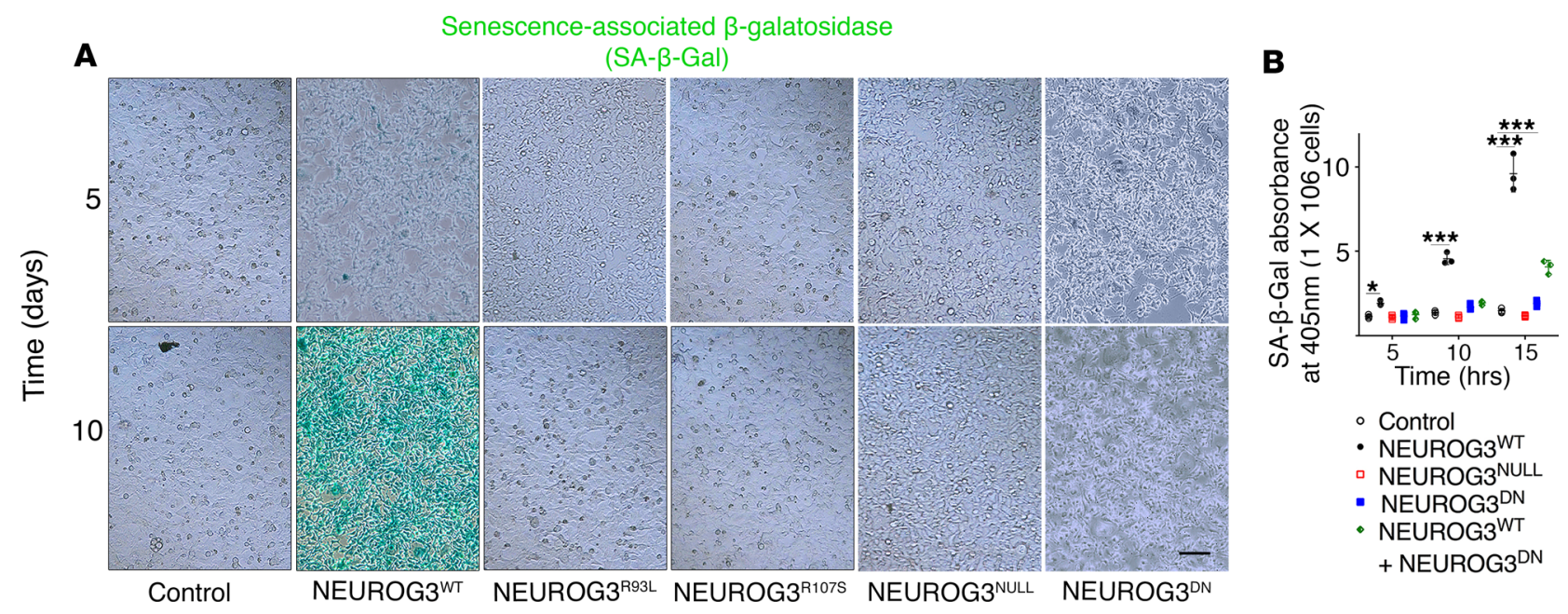

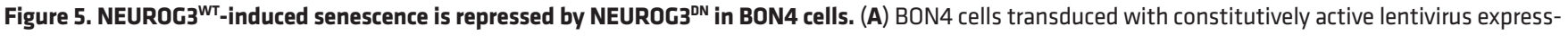
ing NEUROC3 variants and SA $\beta$-Gal staining was performed 5 and 10 days later. Scale bar: $100 \mu \mathrm{m}$. (B) Similarly, SA $\beta$-Gal activity in aqueous phase was assessed 5, 10, and 15 days after transduction of various NEUROC3 variants. ${ }^{*} P<0.05$, ${ }^{* * *} P<0.005,2$-way ANOVA and 2-way Tukey's multiple comparisons.

entirely devoid of pancreatic $\beta$ cells and EECs $(38,39)$, demonstrating that in mice Neurog3 is essential for generation of both pancreatic and intestinal endocrine cells (30-32).

Thus, it is well established that Neurog 3 is required and sufficient for endocrine cell development in the intestines and pancreases of mice and, more recently, pigs (12). Human EEC biology is similarly dependent on NEUROG3; however, our results indicate that it is likely that NEUROG3's role in human pancreatic endocrine development is more complex and may be redundant with other factor(s). Since the age of onset of diabetic symptoms varies so widely, even in patients with identical NEUROG3 genotypes, our findings indicate that the environment, epigenetics, and/or modifier genes influence pancreatic endocrine development in patients with severe NEUROG3 mutations.

\section{Methods}

Genomic DNA isolation and sequencing. Genomic DNA was extracted from saliva by standard procedures and measured by Qubit (Invitrogen). The mRNA of NEUROG3 in encoded on a single exon, and the oligonucleotides and PCR conditions used to amplify the coding region were previously described (ref. 5 and Supplemental Table 2).

Histopathology. Endoscopic formalin-fixed, paraffin-embedded biopsies from the small intestine of the index case were processed using standard techniques and stained for hematoxylin and eosin and immunohistochemical assessment with an anti-chromogranin A antibody (Santa Cruz).

Cloning and site-directed mutagenesis. Expression vectors containing the previously described human NEUROG3 expression vector were used for these studies (5). These vectors also served as the template for the various other constructs used in this study (Supplemental Table 2).

Lentiviral production and transduction. Vectors were constructed by insertion into the multiple cloning site of a third-generation, self-inactivating LV lentivirus-based vector (RRL.sin.cPPT.MCS.IRES.GFP) and produced at the UCLA Core Vector for the production of lentivirus (Supplemental Table 2). All the constructs contain a carboxy terminal FLAG tag, as shown in Figure 1B. Virus titers were determined by HIV1 p24 ELISA (Perkin Elmer) and expressed as p24 equivalents units. Transduction was performed on $10^{5}$ cells with $8 \mu \mathrm{g} / \mathrm{ml}$ polybrene (MilliporeSigma) at $37^{\circ} \mathrm{C}$.

Cell proliferation analysis by MTT and FACS. Cell proliferation was assessed by MTT assay per the manufacturer's instructions (ATCC). BON4 cells were seeded in 12-well flat-bottom well plates (Corning) at a density of $5 \times 10^{5}$ cells per well. Proliferation was also assessed by FACS analysis using $10^{6}$ cells that were harvested and washed with PBS twice, followed by fixation with 4\% PFA for an hour. Fixated cells were washed with $3 \%$ BSA/PBS twice and resuspended in PBS containing $50 \mu \mathrm{g} / \mathrm{ml}$ propidium iodine and 10 $\mu \mathrm{g} / \mathrm{ml} \mathrm{RNaseA}$ for 30 minutes at $25^{\circ} \mathrm{C}$ in the dark. Cells were assessed for DNA content by FACS and analyzed using Cell Quest software (BD). 
A
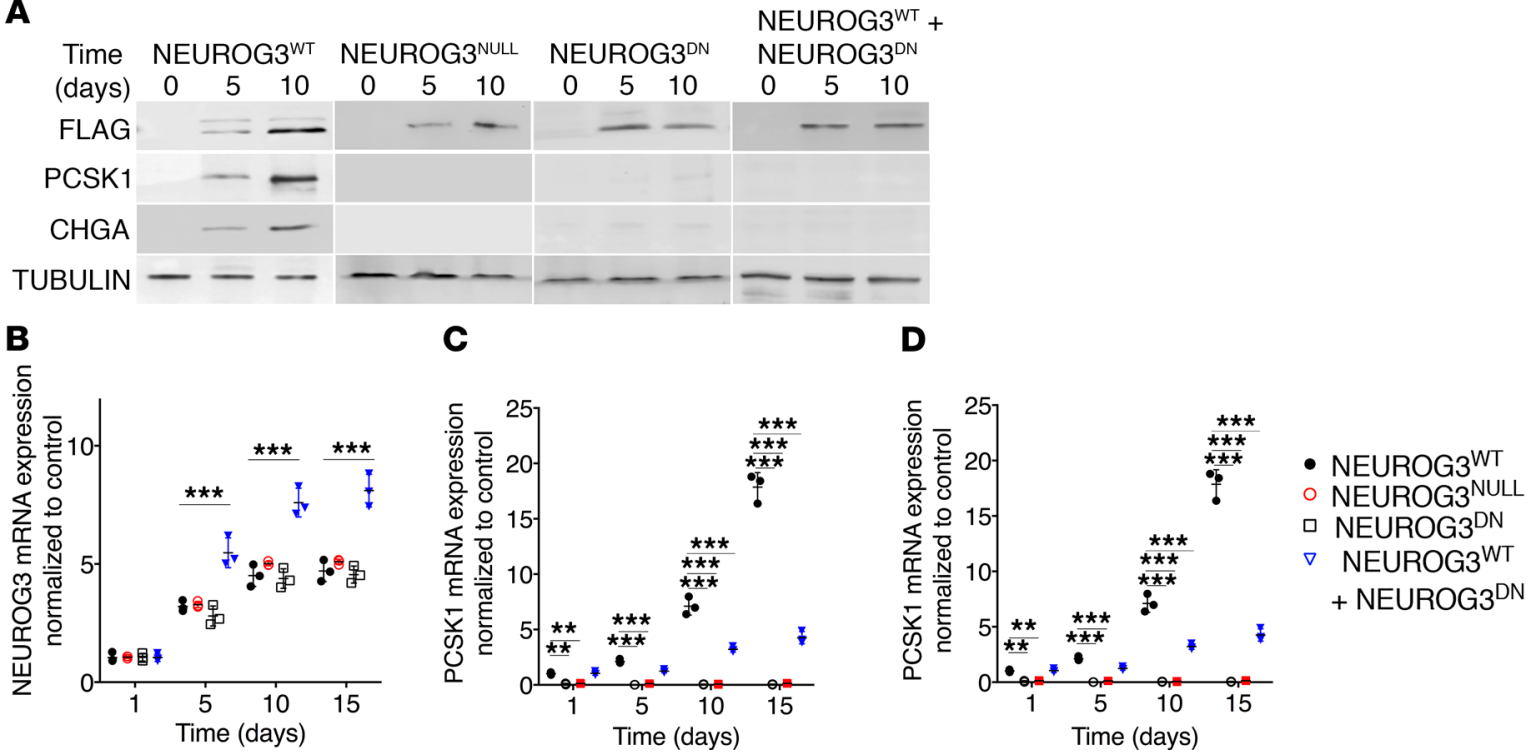

Figure 6. NEUROG $3^{\mathrm{NULL}}$ fails to induce downstream endocrine markers in BON4 cells, and NEUROG ${ }^{\mathrm{ON}}$ attenuates NEUROC ${ }^{\mathrm{WT}}$-induced changes, while retaining marginal activity. (A) Western blot of cellular extracts transduced several days earlier with lentiviruses expressing NEUROC3WT, NEUROC $3^{\text {DN }}$, or both and assessed with anti-FLAG, CHGA, and PCSK1 antibodies. (B-D) Similarly, mRNA levels of various endocrine markers were assessed at the corresponding times after transduction. ${ }^{*} P<0.05,{ }^{*} P<0.01,{ }^{* *} P<0.005,2$-way ANOVA and 2-way Tukey's multiple comparisons.

TUNEL assay. Apoptosis of BON4 cells transduced with NEUROG3 and NEUROG3 mutants was assessed by TUNEL assay (Roche). BON4 cells growth on glass coverslips were fixed in $4 \%$ phosphate-buffered paraformaldehyde for 15 minutes and then washed 3 times with $1 \times$ PBS and $0.3 \%$ Triton X-100 in PBS for 15 minutes. After additional washes, cells were treated with the TUNEL enzyme solution and incubated for 1 hour in a dark, humidified chamber, and washed with PBS, and converter solution was applied. They were then incubated for 30 minutes in a dark, humidified chamber and washed with PBS. Cells were then stained with a Slowfade gold with DAPI (Invitrogen), and covered with a glass slide. As a negative control, TUNEL enzyme was omitted for some reactions.

Quantitative PCR. Steady-state mRNA levels were assessed by quantitative PCR (qPCR) performed with cDNA synthesized from $5 \mu \mathrm{g}$ total RNA; this was performed using TaqMan assay (Life Technology) or Perfecta SYBR green fast mix low rox (QUANTA Biosciences) in a real-time thermocycler (Applied Biosystem, 7500). The results obtained for each individual gene were normalized to the $\beta$-tubulin. The primers used in this work were designed for Primer3, or as suggested by TaqMan, and sequences are shown in Supplemental Table 3.

Luciferase reporter assay. BON cells were plated in 6-well plates at a density of $5 \times 10^{5}$ cells per well in DMEM-F12 media supplemented with 10\% FBS and penicillin/streptomycin. Depending on the experiment, cells were traduced with 1 of several lentiviruses as described earlier. One day later, the cells were transfected with 1 pmol of the pGL3 Basic (Promega) plasmid containing the promoter areas of human NEUROD1 $(1 \mathrm{~Kb})(5)$, or p21 (-592/141), or SLC5A1 (-5370/+22) (ref. 17 and Supplemental Table 2) using the lipofectamine reagent (Invitrogen). Two days later, cell lysates and luciferase levels were measured with the dual luciferase assay kit (Promega). Luciferase levels were normalized by renilla luciferase. Data are showed as mean \pm SEM over PGL3-B control.

$S A \beta$-Gal staining. SA $\beta$-Gal staining was used to determine replicative senescence as previously described (20). Cells plated in 6-well plates were washed twice with PBS and fixed with 4\% PFA. They were then washed twice with PBS by gently shaking, and $1 \mathrm{ml} \mathrm{SA} \beta$-Gal staining solution was added and incubated at $37^{\circ} \mathrm{C}$. Cells were observed every 4 hours for 12 hours and then every 12 hours. The optimal incubation period was determine based on the visibility of stained cells in the test sample but not in the control sample. Then, cells were lysed with $200 \mu \mathrm{l}$ cell culture lysis reagent 5X (Promega), and the absorbance was measured at $405 \mathrm{~nm}$ on a VERSAmax microplate reader (Molecular Devices).

Western blot analysis. Cells were lysed with RIPA lysis buffer, scraped into microfuge tubes, incubated 5 minutes at $25^{\circ} \mathrm{C}$, and then centrifuged at max speed at $4^{\circ} \mathrm{C}$ for 10 minutes. Concentration of the proteins 
A

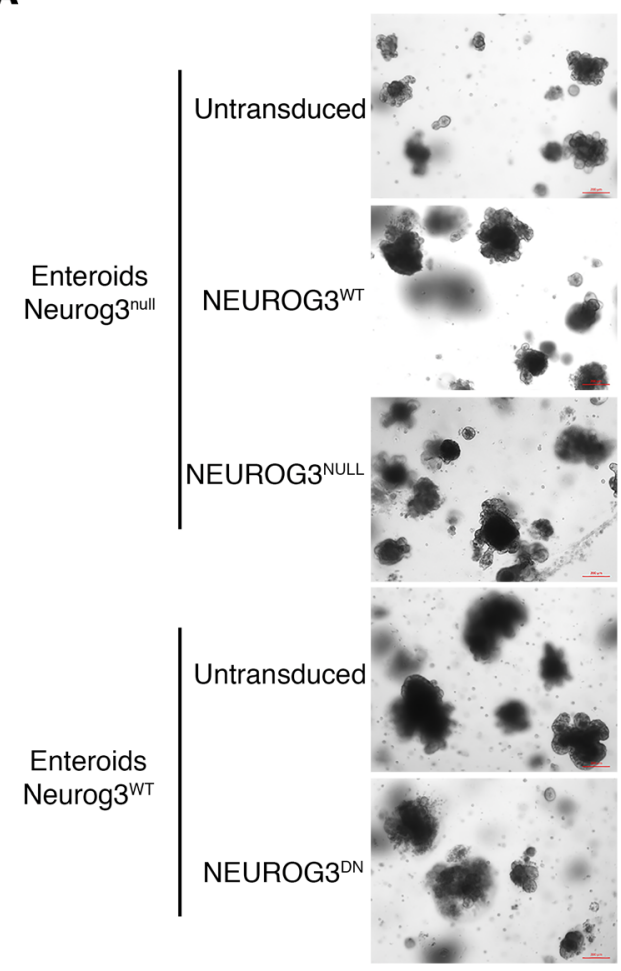

B

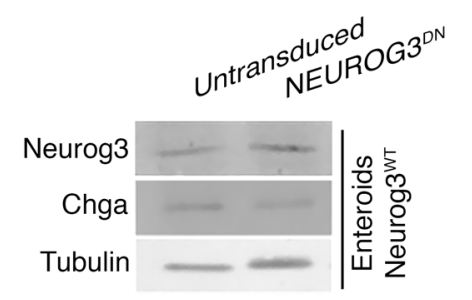

C

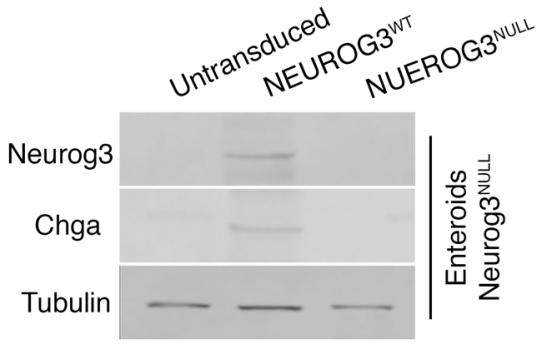

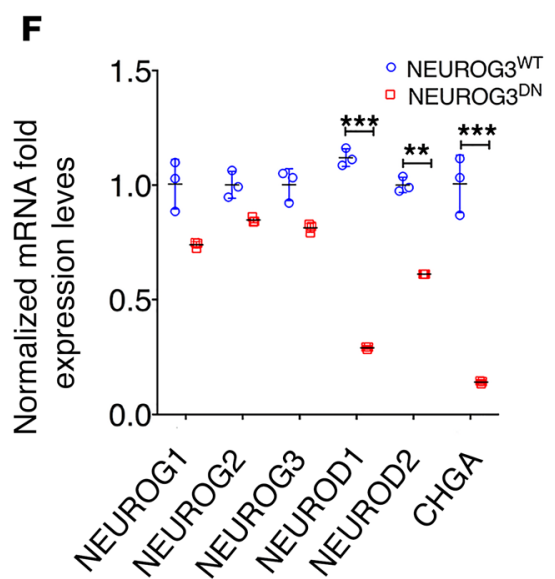

G

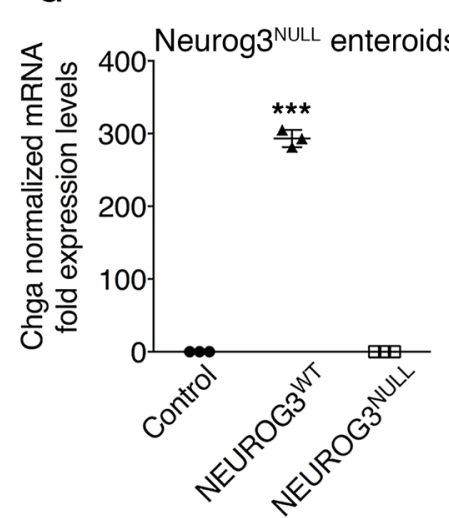

H

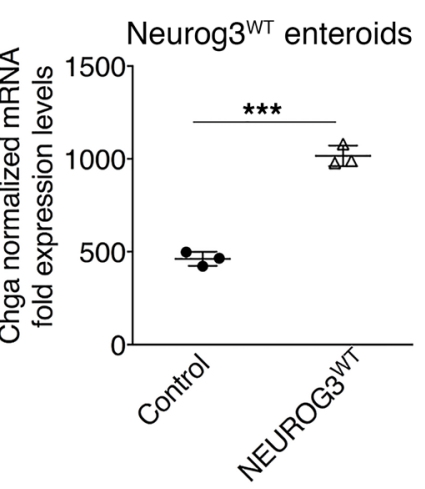

I

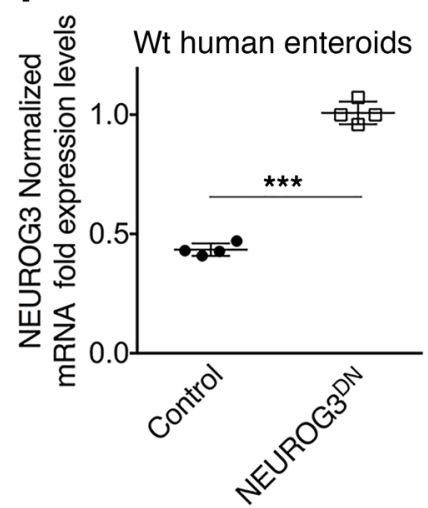

J

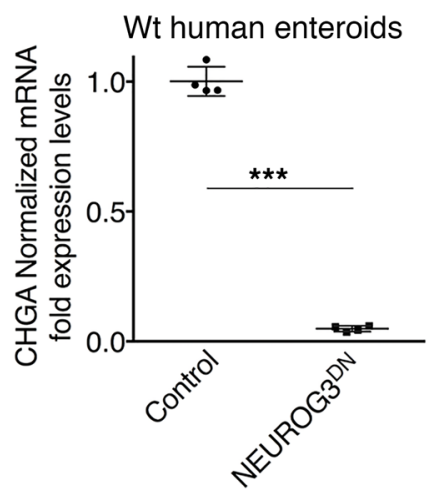

Figure 7. Assessment of endocrine cell induction in enteroids from Neurog $3^{\mathrm{WT}}$ or Neurog $3^{\text {null }}$ mice or human intestines from normal and NEUROG $3^{\mathrm{DN}}$

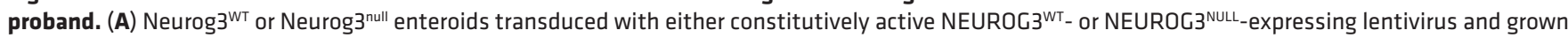
in culture for 7 days. (B and C) Western blot of enteroids cell extracts isolated from (B) Neurog $3^{{ }^{W T}}$ or (C) Neurog3 ${ }^{\text {null }}$ mice. Enteroids were untransduced or transduced with NEUROC $3^{\text {WT }}$ or NEUROG3 ${ }^{\text {NULL }}$ and assessed for proteins using anti-Neurog3, Chga and tubulin antibodies. (D) Western blot of enteroids cell extracts isolated from a normal human (WT) subject untransduced or transduced with NEUROC $3^{\mathrm{DN}}$ and assessed 7 days later for proteins. (E) Human enteroids from WT and NEUROG $3^{\mathrm{DN}}$ subjects were examined for specified proteins and (F) mRNA. (G and $\left.\mathbf{H}\right)$ mRNA isolated from murine (C) Neurog $3^{\text {NULL }}$ and (H) Neurog $3^{\text {WT }}$ enteroids transduced with NEUROC3 ${ }^{\text {WT }}$ or NEUROG3 ${ }^{\text {NULL }}$ and WT enteroids transduced with NEUROG $3^{\text {WT }}$ and assessed 7 days later. (I and J) mRNA isolated from WT human enteroids and transduced with NEUROG $3^{\mathrm{DN}}$ and assessed 7 days later. $(\mathbf{F}-\mathrm{J}){ }^{*} P<0.05,{ }^{* *} P<0.01,{ }^{* *} P<0.005$, 1-way ANOVA and Dunnett's multiple comparison. (G-J) ${ }^{*} P<0.05$, ${ }^{* *} P<0.01,{ }^{* *} P<0.005$, unpaired Student's $t$ test.

was determined using the Qubit protein assay (Life Technologies). Protein (10 g) was loaded onto a 10\% polyacrylamide gel and subjected to SDS-PAGE and blotted onto immune blot Amersham Hybond 0.2 PVDF (GE Healthcare Life Sciences). Membranes were blotted using standard procedures using the primary antibodies shown in Supplemental Table 4. Secondary antibodies used were either an ECL anti-mouse IgG-HRP-linked whole antibody or anti-rabbit IgG-HRP-linked whole antibody. Western blots were visualized using the ECL plus Western blotting kit (GE Healthcare) and scanned on a Thyphoon 9410 imager (GE Healthcare) using antibodies shown in Supplemental Table 4. 
Microscopy and staining. BON4 cells transduced with NEUROG3 or NEUROG $3^{D N}$ were grown on glass cover slips and fixed in 4\% PFA for 15 minutes. They were then washed 3 times with $1 \times$ PBS and treated with blocking buffer ( $1 \times$ PBS/5\% normal serum/0.3\% Triton X-100) for 60 minutes and washed 3 times with PBS for 2 minutes, incubated with diluted primary antibody (Supplemental Table 4) for 2 hours at room temperature, and rinsed 3 times in $1 \times$ PBS for 5 minutes each. They were then incubated in fluorochrome-conjugated secondary antibody diluted in antibody dilution buffer ( $1 \times$ PBS $/ 1 \%$ BSA $/ 0.3 \%$ Triton $\mathrm{X}-100$ ) for 1-2 hours at room temperature in the dark, rinsed 3 times in $1 \times$ PBS for 5 minutes each, and mounted using Gold Antifade Reagent with DAPI (Invitrogen).

Generation of BON4 cells. The BON4 cell line used here was generated in-house from the BON cells that were obtained from ATCC. The BON4 cells were generated from the parental BON cell that had a more heterogeneous appearance and growth characteristics. These cells were maintained in DMEM-F12 media supplemented with $10 \%$ FBS.

Murine enteroid isolation, propagation, and transduction method. Small intestinal crypts were isolated from Neurog3-null (Neurog $3^{\text {null}}$ ) pups immediately after birth, using a previously described protocol (40). Crypts were initially resuspended in $5 \mathrm{~mL}$ Basic Crypt Media (BCM) consisting of Advanced DMEM/Ham's F12 (Invitrogen) with $1 \times$ Antibiotic-Antimycotic, $2 \mathrm{mM}$ Glutamax (Invitrogen), and $10 \mathrm{mM}$ HEPES (Invitrogen). Aliquots were microcentrifuged to yield crypts pellets for culture purposes.

Human enteroid isolation, propagation, and transduction method. Duodenal crypts were obtained and grown as enteroids using previously described methods. In general, enteroids were grown in 3D culture with Matrigel (Corning) and $1 \mathrm{mM} \mathrm{N}$-Acetylcysteine (MilliporeSigma), $100 \mathrm{ng} / \mathrm{ml}$ recombinant murine Noggin (PeproTech), $50 \mathrm{ng} / \mathrm{ml}$ recombinant murine EGF (PeproTech), N2 supplement (Invitrogen), B27 supplement (Invitrogen), and $1 \mu \mathrm{g} / \mathrm{ml}$ recombinant human R-spondin1 (R\&D Systems), $10 \mu \mathrm{M}$ ROCK inhibitor (MilliporeSigma), and $5 \mu \mathrm{M}$ GSK inhibitor (Stemgent).

Statistics. For multiple comparisons, 1- or 2-way ANOVA was followed by Tukey's or Dunnett's multiple-comparisons test. For comparisons involving 1 independent variable with 2 groups, we performed 2-tailed Student's $t$ tests. $P$ values were considered significant at $P<0.05$. Variation is reported as SEM.

Study approval. Discarded human intestinal epithelium was obtained with approval from the UCLA Human Investigational Review Board at the UCLA Department of Pathology, as previously described (41). The index cases and their families were enrolled in the UCLA pediatric diarrhea study that was approved by the UCLA institutional review board. Informed consent was obtained from members of both families. Pertinent medical and pathology records were obtained and summarized for the patients.

\section{Author contributions}

RSSV, MGM, MB, and MGGC conceived and designed the experiments. RSSV, JW, MB, HC, and SG performed the experiments. RSSV, MGM, MB, and HC analyzed the data. MGGC and PP contributed reagents, materials, and/or analysis tools. SVW and MSG performed experiments and analyzed the data. MGM, MB, and SG wrote the paper. RSSV, MGGC, MB, HC, and SG critically reviewed the manuscript.

\section{Acknowledgments}

Research reported in this publication was supported by the National Institute of Diabetes and Digestive and Kidney Disorders (R01DK083762; DK41301; DK118640) and the California Institute of Regenerative Medicine (RT2-01985).

Address correspondence to: Martín G. Martín, David Geffen School of Medicine, UCLA, 10833 Le Conte Avenue, Los Angeles, California 90095, USA. Phone: 310.794.5532; Email: mmartin@mednet.ucla.edu. Or to: Senta Georgia, Department of Pediatrics, Division of Endocrinology, Children's Hospital of Los Angeles, USC, 4650 Sunset Boulevard, Los Angeles, California 90027, USA.

1. Watada H, Scheel DW, Leung J, German MS. Distinct gene expression programs function in progenitor and mature islet cells. $J$ Biol Chem. 2003;278(19):17130-17140.

2. Bjerknes M, Cheng H. Neurogenin 3 and the enteroendocrine cell lineage in the adult mouse small intestinal epithelium. Dev Biol. 2006;300(2):722-735.

3. Gradwohl G, Dierich A, LeMeur M, Guillemot F. Neurogenin3 is required for the development of the four endocrine cell lineages of the pancreas. Proc Natl Acad Sci USA. 2000;97(4):1607-1611.

4. Martín MG, et al. Congenital proprotein convertase 1/3 deficiency causes malabsorptive diarrhea and other endocrinopathies in 
a pediatric cohort. Gastroenterology. 2013;145(1):138-148.

5. Wang J, et al. Mutant neurogenin-3 in congenital malabsorptive diarrhea. N Engl J Med. 2006;355(3):270-280.

6. Thiagarajah JR, et al. Advances in evaluation of chronic diarrhea in infants. Gastroenterology. 2018;154(8):2045-2059.e6.

7. Rubio-Cabezas O, Gómez JL, Gleisner A, Hattersley AT, Codner E. Hypogonadotropic hypogonadism and short stature in patients with diabetes due to neurogenin 3 deficiency. J Clin Endocrinol Metab. 2016;101(10):3555-3558.

8. Rubio-Cabezas O, Codner E, Flanagan SE, Gómez JL, Ellard S, Hattersley AT. Neurogenin 3 is important but not essential for pancreatic islet development in humans. Diabetologia. 2014;57(11):2421-2424.

9. Rubio-Cabezas O, et al. Permanent neonatal diabetes and enteric anendocrinosis associated with biallelic mutations in NEUROG3. Diabetes. 2011;60(4):1349-1353.

10. Zhu Z, et al. Genome editing of lineage determinants in human pluripotent stem cells reveals mechanisms of pancreatic development and diabetes. Cell Stem Cell. 2016;18(6):755-768.

11. McGrath PS, Watson CL, Ingram C, Helmrath MA, Wells JM. The basic helix-loop-helix transcription factor NEUROG3 is required for development of the human endocrine pancreas. Diabetes. 2015;64(7):2497-2505.

12. Sheets TP, et al. Targeted mutation of NGN3 gene disrupts pancreatic endocrine cell development in pigs. Sci Rep. 2018;8(1):3582.

13. Krentz NAJ, et al. Phosphorylation of NEUROG3 links endocrine differentiation to the cell cycle in pancreatic progenitors. Dev Cell. 2017;41(2):129-142.e6.

14. Solorzano-Vargas RS, et al. The cellular regulators PTEN and BMI1 help mediate NEUROGENIN-3-induced cell cycle arrest. J Biol Chem. 2019;294(41):15182-15192.

15. Cortina G, et al. Enteroendocrine cell dysgenesis and malabsorption, a histopathologic and immunohistochemical characterization. Hum Pathol. 2007;38(4):570-580.

16. Huang HP, Liu M, El-Hodiri HM, Chu K, Jamrich M, Tsai MJ. Regulation of the pancreatic islet-specific gene BETA2 (neuroD) by neurogenin 3. Mol Cell Biol. 2000;20(9):3292-3307.

17. Martín MG, Wang J, Solorzano-Vargas RS, Lam JT, Turk E, Wright EM. Regulation of the human Na(+)-glucose cotransporter gene, SGLT1, by HNF-1 and Sp1. Am J Physiol Gastrointest Liver Physiol. 2000;278(4):G591-G603.

18. Hollenberg SM, Sternglanz R, Cheng PF, Weintraub H. Identification of a new family of tissue-specific basic helix-loop-helix proteins with a two-hybrid system. Mol Cell Biol. 1995;15(7):3813-3822.

19. Miyatsuka T, Kosaka Y, Kim H, German MS. Neurogenin3 inhibits proliferation in endocrine progenitors by inducing Cdkn1a. Proc Natl Acad Sci USA. 2011;108(1):185-190.

20. Itahana K, Itahana Y, Dimri GP. Colorimetric detection of senescence-associated $\beta$ galactosidase. Methods Mol Biol. 2013;965:143-156.

21. Wang $\mathrm{F}$, et al. Isolation and characterization of intestinal stem cells based on surface marker combinations and colony-formation assay. Gastroenterology. 2013;145(2):383-95.e1.

22. Lahar N, et al. Intestinal subepithelial myofibroblasts support in vitro and in vivo growth of human small intestinal epithelium. PLoS One. 2011;6(11):e26898.

23. Russo L, et al. Permanent diabetes during the first year of life: multiple gene screening in 54 patients. Diabetologia. 2011;54(7):1693-1701

24. Jensen JN, Rosenberg LC, Hecksher-Sørensen J, Serup P. Mutant neurogenin-3 in congenital malabsorptive diarrhea. $N$ Engl $J$ Med. 2007;356(17):1781-1782.

25. Pinney SE, et al. Neonatal diabetes and congenital malabsorptive diarrhea attributable to a novel mutation in the human neurogenin-3 gene coding sequence. J Clin Endocrinol Metab. 2011;96(7):1960-1965.

26. Smith SB, et al. Rfx6 directs islet formation and insulin production in mice and humans. Nature. 2010;463(7282):775-780.

27. Soyer J, et al. Rfx6 is an Ngn3-dependent winged helix transcription factor required for pancreatic islet cell development. Development. 2010;137(2):203-212.

28. Pauerstein PT, et al. Dissecting human gene functions regulating islet development with targeted gene transduction. Diabetes. 2015;64(8):3037-3049.

29. Brezar V, Carel JC, Boitard C, Mallone R. Beyond the hormone: insulin as an autoimmune target in type 1 diabetes. Endocr Rev. 2011;32(5):623-669.

30. Faideau B, et al. Tolerance to proinsulin-2 is due to radioresistant thymic cells. J Immunol. 2006;177(1):53-60.

31. Falcone M, Lee J, Patstone G, Yeung B, Sarvetnick N. B lymphocytes are crucial antigen-presenting cells in the pathogenic autoimmune response to GAD65 antigen in nonobese diabetic mice. J Immunol. 1998;161(3):1163-1168.

32. De Meyts P. Insulin and its receptor: structure, function and evolution. Bioessays. 2004;26(12):1351-1362.

33. Kurosaki T, Popp MW, Maquat LE. Quality and quantity control of gene expression by nonsense-mediated mRNA decay. Nat Rev Mol Cell Biol. 2019;20(7):406-420.

34. Hsu MK, Lin HY, Chen FC. NMD Classifier: A reliable and systematic classification tool for nonsense-mediated decay events. PLoS One. 2017;12(4):e0174798.

35. Villasenor A, Chong DC, Cleaver O. Biphasic Ngn3 expression in the developing pancreas. Dev Dyn. 2008;237(11):3270-3279.

36. Johansson KA, et al. Temporal control of neurogenin 3 activity in pancreas progenitors reveals competence windows for the generation of different endocrine cell types. Dev Cell. 2007;12(3):457-465.

37. Murtaugh LC. Pancreas and beta-cell development: from the actual to the possible. Development. 2007;134(3):427-438

38. Jensen J, et al. Control of endodermal endocrine development by Hes-1. Nat Genet. 2000;24(1):36-44.

39. Gu G, Dubauskaite J, Melton DA. Direct evidence for the pancreatic lineage: NGN3+ cells are islet progenitors and are distinct from duct progenitors. Development. 2002;129(10):2447-2457.

40. Lei NY, et al. Intestinal subepithelial myofibroblasts support the growth of intestinal epithelial stem cells. PLoS One. 2014;9(1):e84651.

41. Rouch JD, et al. Development of functional microfold (M) cells from intestinal stem cells in primary human enteroids. PLoS One. 2016;11(1):e0148216. 